\title{
Could PD-L1 prove to be an effective therapeutic target for bladder cancer?
}

\section{"The last significant advance in metastatic bladder cancer treatment came with the application of cisplatin-based chemotherapies over two decades ago."}

Keywords: immune checkpoint inhibition • urothelial bladder cancer

Highlighting this years' American Society for Clinical Oncology (ASCO) conference were presentations focusing on T-cell immune checkpoint blockade as a therapeutic target in solid tumors. Promising results in melanoma, lung and renal cancers have led to further disease-specific Phase I and II trials of immune checkpoint blockade in other solid tumors refractory to treatment.

Metastatic urothelial bladder cancer (UBC) is one such tumor; this disease is associated with limited treatment options and a poor prognosis. The last significant advance in metastatic bladder cancer treatment came with the application of cisplatin-based chemotherapies over two decades ago. Despite the widespread use of cisplatin-based combination regimens and improved supportive care, 5 year survival rates for metastatic UBC remain around $15 \%$. The response rates with second-line chemotherapies continue to be dismal, generally around $20 \%$ or less, with median survivals of 6-9 months. Thus, the identification of new therapeutic targets for $\mathrm{UBC}$ is crucial to improving outcomes in this disease. There is already precedent for use of immune therapy in UBC; treatment of superficial bladder cancer with intravesicular BCG remains the standard of care for non-muscle invasive carcinoma. BCG stimulates a plethora of immune responses, including the activation and recruitment of $\mathrm{CD}^{+}$and $\mathrm{CD} 8{ }^{+} \mathrm{T}$ lymphocytes.
The programmed death-1 receptor is an inhibitory transmembrane receptor expressed by $\mathrm{CD}^{+}$and $\mathrm{CD} 8^{+} \mathrm{T}$ lymphocytes that plays a crucial role in the regulation of T-cell response. It is a member of the CD28/CTLA-4 family of T-cell regulators that, when engaged by its ligands, PD-L1 (B7-H1) or PD-L2 (B7-DC), leads to dephosphorylation of proximal signaling molecules downstream of the TCR complex via src homology region 2 domain-containing phosphatase (SHP)-1 and -2, as well as augmentation of PTEN, leading to decreased T-cell proliferation, survival and protein synthesis. Ligand expression in the form of PD-L1 and PD-L2 is, thus, necessary in the development and maintenance of immune tolerance and the prevention of autoimmunity. The upregulation of PD-L1 expression by tumor cells, however, is thought to be a mechanism by which solid tumors evade or develop tolerance to immune regulation. Urothelial bladder tumors have been shown to have levels of PD-L1 expression similar to other solid tumors [1]. Interestingly, higher levels of PD-L1 expression are seen in more advanced and metastatic tumors compared with earlystage tumors [2], both supporting the notion the that higher PD-L1 expression can help tumors evade immune regulation, as well as highlighting the promise of PD-L1 blockade.

At ASCO, updated results of an ongoing Phase I study of the human anti-PD-L1 monoclonal antibody MPDL3290A in meta-
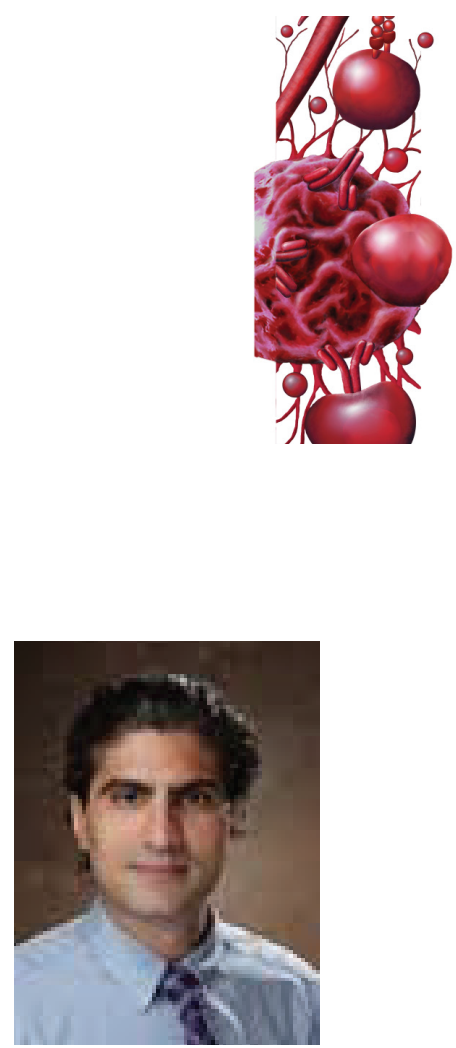

Navid Hafez

Yale Comprehensive Cancer Center, Yale School of Medicine, 333 Cedar Street, Fitkin 3, New Haven, CT 06520-8028, USA

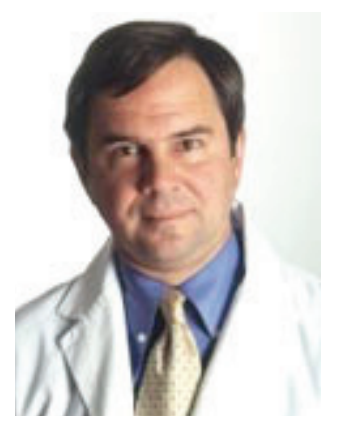

Daniel P Petrylak

Author for correspondence:

Genitourinary Oncology Research Program, Signal Transduction Program, Yale Comprehensive Cancer Center, Yale School of Medicine, 333 Cedar Street, Fitkin 3, New Haven, CT 06520-8028, USA Tel.: +1 2037853597 daniel.petrylak@yale.edu 
static UBC were presented [3]. An overall response rate of $52 \%$ was observed in 65 patients treated at $15 \mathrm{mg} / \mathrm{kg}$ intravenous every 3 weeks. Nearly all (97\%) of these patient were previously treated with cisplatin-based chemotherapy, the vast majority of which $(71 \%)$ had one or more prior regimens. Of the 30 patients who had strong (2+ to $3+)$ PD-L1 expression by immunohistochemistry (IHC), 43\% demonstrated an objective response. In the 25 patients with weak IHC ( 0 to $1+$ ) to PDL-1, 11\% demonstrated an objective response. The median time to first response was 42 days (range 38-85 days). At the time of the data analysis, 16 of the 17 responds were ongoing. The predominant toxicity of patients treated with MPDL3290A was fatigue.

\section{"MPDL3290A was given Breakthrough Therapy \\ Designation by the US FDA on 31 May 2014.}

These results identify immune checkpoint regulation via PD-1/PD-L1 blockade as a promising therapeuticlead in metastatic urothelial cancer. In fact, MPDL3290A

\section{References}

1 Zhou W, Chen L. Inhibitory B7-family molecules in the tumour microenvironment. Nat. Rev. Immunol. 8(6), 467-477 (2008).

2 Nakanishi J, Wada Y, Matsumoto K, Azuma M, Kikuchi $\mathrm{K}$, Ueda S. Overexpression of B7-H1 (PD-L1) significantly associates with tumor grade and postoperative prognosis in was given Breakthrough Therapy Designation by the US FDA on 31 May 2014. A recently opened Phase II trial of 300 platinum refractory UBC patients will further define the response rate of MPDL3290A in the population of patients with platinum refractory UBC. Upfront treatment with MPDL3290A will also be examined in patients who are platinum ineligible. That $11 \%$ of patients who had weak or no expression of PDL-1 by IHC responded demonstrates that further studies are needed to correlate biological markers with response and progression.

\section{Financial \& competing interests disclosure}

DP Petrylak is a consultant with Genetech, and has research support from Genetech and Merck. The authors have no other relevant affiliations or financial involvement with any organization or entity with a financial interest in or financial conflict with the subject matter or materials discussed in the manuscript apart from those disclosed.

No writing assistance was utilized in the production of this manuscript.

human urothelial cancers. Cancer Immunol. Immunother. 56(8), 1173-1182 (2007).

3 Powles T, Vogelzang NJ, Fine DG et al. Inhibition of PD-L1 by MPDL3280A and clinical activity in pts with metastatic urothelial bladder cancer (UBC). J. Clin. Oncol. 32(5s Suppl.), Abstract 5011 (2014). 